\title{
Modelo de consumo de drogas entre minorías étnicas en un ambiente comunitario de drogadicción
}

\author{
Hamdy F. Moselhy, MBBCh, Msc, DPM, \\ MRCPsych* \\ Ian Telfer, MBChB, MRCPsych** \\ * Psiquiatra, Centro de Conducta Adictiva, \\ Highcroft Hospital, 71 Fentham Road \\ Erdington, Birmingham, B23 6AL \\ ** Psiquiatra, Psychiatric OPD, Tameside \\ General Hospital, Fountain Street, Ashton \\ Under Lyne, Lancashire, OL6 9RW \\ REINO UNIDO
}

RESUMEN - El objetivo de este estudio fue el de examinar el modelo de abuso de tóxicos que seguían ciertas minorías étnicas al compararlas con un grupo de personas de nacionalidad británica. A través de una recopilación retrospectiva nos encargamos de revisar a todos los pacientes que fueron valorados en un periodo de 3 años. 179 de ellos cumplieron los requisitos para ser incluidos en el estudio. Se examinó a 140 pacientes caucasianos, entre los que se encontraban 119 británicos, 18 irlandeses y otros 3 europeos; también participaron en el estudio 24 pacientes asiáticos y 15 afro-caribeños. El estilo de abuso de tóxicos evidentemente, era diferente cuando comparábamos cada minoría étnica en relación con el grupo británico. Se apreciaba mayor proporción de consumo de opio en la muestra asiática que en la británica. En cambio, el grupo de afro-caribeños era el mayor consumidor de cocaína. También se registraron importantes consumos de opio entre el grupo irlandés; además, comparado con el grupo británico, el 100\% de sus miembros compartía las jeringuillas.

Estos hallazgos demuestran las diferentes formas de consumir tóxicos que tienen las minorías étnicas. Incluso entre los mismos grupos raciales, por ejemplo en los caucásicos, el modelo de consumo es diferente. Por todo ello son necesarios Servicios orientados hacia las diferencias que presentan las diversas etnias en lo que al abuso de tóxicos se refiere. 


\section{Introducción}

El término "etnicidad" define a los individuos que se consideran a sí mismos, o son considerados por los demás como poseedores de unas mismas características y que a la vez les diferencian de otros colectivos de la sociedad, dentro de la cual se comportan de una manera específica. El término se acuñó como opuesto al de "raza" ya que, aunque los miembros de un grupo étnico pueden ser identificados según sus atributos raciales, también pueden compartir otras características culturales como religión, empleo, idioma o política. También es importante establecer las diferencias entre grupos étnicos y clases sociales, pues el hecho de pertenecer a uno de estos colectivos determina la estratificación de la sociedad, incluyendo a individuos que comparten (o se supone que comparten) características comunes que sustituyen a la clase social (Marshall 1996). No se debe entender que una cultura étnica es una entidad fija y estática. La "etnicidad" no es sólo lo que la gente cree ser -o como se identifican- ya que también es una forma de clasificar por otros grupos a diferentes colectivos dentro de una sociedad multicultural. De este modo, conceptos como la identificación étnica, la cultura étnica y los límites dentro de los cuales se enmarca un grupo étnico pueden cambiar y evolucionar con el tiempo, según las fuerzas sociales, económicas y políticas que imperan en la sociedad (Lieberson 1985).

El papel que juega la "etnicidad" en la etiología y en los resultados de una enfermedad psiquiátrica ha sido ampliamente reconocido (Cochran 1977, Karon et al. 1987, Birchwood et al. 1992).

Los estudios más recientes han demostrado cómo la "etnicidad" puede mejorar nuestra comprensión acerca de la naturaleza y la etiología de los trastornos por abuso de sustancias
(HWU et al. 1992), así como sobre los modelos de presentación de estos desórdenes (McKeigue et al. 1993). También puede informar sobre las decisiones que se toman en cuanto al diseño y a la puesta en práctica de programas de prevención que sean culturalmente específicos (Catalano et al. 1993).

A pesar de las ventajas que la investigación intercultural ha aportado en Norteamérica, pocos autores han estudiado de forma simultánea las diferencias étnicas entre los consumidores de drogas en el Reino Unido.

Los datos oficiales y los aportados por los drogadictos (Cheung 1990) dan prueba de que existen variaciones étnicas y raciales en cuanto al consumo de drogas. Los estudios oficiales utilizan los datos propios para seleccionar muestras de adictos reconocidos como objeto de estudio. Las estadísticas de tipo criminal/penal, tales como las que posee la policía o las específicamente judiciales contienen elementos étnicos o raciales relacionados con el consumo de drogas de los delincuentes, muchos de los cuales probablemente son adictos. Los datos procedentes de pacientes con que cuentan instituciones tales como hospitales y centros de tratamiento de toxicómanos proporcionan información importante sobre estos aspectos raciales o étnicos propios de los adictos así diagnosticados.

Los estudios que han utilizado muestras de adictos bien definidos ocupan una parte importante en el conjunto de las investigaciones relacionadas con la "etnicidad" y con el consumo de drogas. Por ejemplo, un estudio realizado en italianos que se inyectaban heroína intravenosa y desarrollado en Londres por Lipsedge et al. (Lipsedge et al. 1993) mostró que el 30\% eran VIH positivo, que su comportamiento era arriesgado y que tenían un acceso limitado a los servicios. En el estudio de O'Brien y Power (O’Brien \& Power 1998) sobre los consumidores en Gran Bretaña, concluyeron que la proporción de drogas más baratas y accesi- 
bles estaban disponibles en este país. También informaron que los cambios personalmente reportados sobre el consumo de drogas después de la migración incluían un aumento en su consumo, así como la utilización de la via parenteral y el compartir agujas y jeringuillas. La soledad y el aislamiento son las razones debido a las cuales, sufrieron recaídas. Las mujeres que fueron entrevistadas echaban de menos la vida en comunidad propia de Irlanda y buscaban nuevos contactos fuera de casa, muchos de los cuales resultó que eran consumidores. En muchos casos el consumo de drogas en Inglaterra era bastante más 'caótico' y mas difícil de controlar que puede suceder en Irlanda. Shaffer et al. 1985, realizaron entrevistas a 354 adictos a narcóticos del sexo masculino seleccionados al azar a partir de los datos aportados por estudios anteriores sobre personas adictas que habían sido detenidas por el Departamento de Policía de Baltimore entre 1952 y 1976. Descubrieron que la utilización de drogas no-narcóticas estaba muy extendida entre los adictos a narcóticos de raza blanca y entre los de raza negra. La utilización de cocaína mantiene una relación más estrecha con el aumento de la actividad criminal entre los negros que entre los blancos. Los estudios actuales (Anglin et al. 1988a, Anglin et al. 1988b) en muestras de pacientes ingleses y chicanos que habían sido admitidos en tres programas de mantenimiento con Metadona en California, mostrando como existe una relación directa entre consumo de drogas y nivel de criminalidad entre ambos grupos étnicos.

Otro conjunto de estudios diseña sus muestras, generalmente a partir de poblaciones no institucionalizadas, y utilizando el método de la propia confesión personal para medir el abuso de drogas. En uno de los informes que integran su amplio estudio, Kandel et al. (1975) descubrieron que los estudiantes de raza blanca acusaban una mayor tendencia a consumir marihuana, hachís, LSD, anfetaminas, barbitúricos, tranquilizantes, narcóticos diferentes de la heroína e inhalantes que lo que sucedía en los negros e hispanos. En cambio, estos últimos consumían más cocaína y heroína.

El hecho de que los estudiantes de raza blanca consuman más drogas que los estudiantes negros, resulta evidente si nos atenemos a los resultados descriptivos del proyecto que cada año lleva a cabo el Instituto de Investigaciones Sociales de la Universidad de Michigan. El objetivo del estudio es el de recoger datos teniendo en cuenta los estilos de vida y los valores de los "seniors" de los Institutos de Segunda Enseñanza americanos (Bachman et al. 1987). Otro de los propósitos de la investigación es el de estudiar los modelos de uso/abuso de drogas entre asiáticos, irlandeses y afro-caribeños, comparándolos con el británico caucásico en el contexto de un equipo comunitario de drogodependientes.

\section{El marco clínico}

El Baker Street Center es un equipo comunitario de drogodependientes que ofrece un servicio de consultas externas a una población de 124.000 personas en la zona oeste de Birmingham. Tiene una política de recepción abierta y los miembros del equipo multidisciplinar ofrecen una amplia variedad de modalidades de tratamiento.

\section{Método}

Se revisaron las historias clínicas de pacientes con trastornos por consumo de drogas que habían sido atendidos por un psiquiatra durante un periodo de tres años (entre mayo de 1992 y mayo de 1995). Los datos demográficos, los referentes al tipo y modelo de uso de drogas, así como los datos 
forenses se recopilaron por medio de un impreso que había sido utilizado durante la valoración de estos individuos. Los criterios de exclusión eran presentar además problemas de alcoholismo y no haber sido examinados por un psiquiatra con el fin de garantizar la validez del diagnóstico.

\section{Material}

Un total de 179 pacientes cumplieron los criterios necesarios para ser incluidos. 139 hombres y 140 mujeres. Se excluyeron 140 caucásicos, 119 británicos, 18 irlandeses y otros 3 europeos, 24 asiáticos y 15 afrocaribeños, en este caso debido a su reducido número. Entre los británicos caucásicos, había 30 mujeres (el 25,2\%) y 89 hombres (el 74,8\%); y en cuanto al grupo de personas de origen asiático se registró a una mujer (el $4,2 \%$ ) y a 23 hombres (el 95,8\%). Entre los irlandeses, había 3 mujeres (el 16,6\%) y 15 hombres (el 83,4\%) y, en cuanto al grupo de los afro-caribeños se refiere, había 5 mujeres $(\mathrm{el} 33,3 \%$ ) y 10 hombres (el 66,7\%).

\section{Resultados}

La tabla I muestra las medias y la desviaciones estándar de la edad de presentación, así como la edad que tenían los individuos la primera vez que consumieron drogas. También presenta variables relativas al consumo de drogas de los cuatro grupos.

Tabla I

\begin{tabular}{|c|c|c|c|c|}
\hline Cau & sico-Británico n (\%) & Asiático n (\%) & Irlandés n (\%) & Afro-Caribeño n $(\%)$ \\
\hline Edad de presentación & $32,2 \pm 7,5$ & $24,5 \pm 4,4$ & $30 \pm 5,5$ & $31,2 \pm 6,8$ \\
\hline Edad del primer consumo & $21,3 \pm 6,1$ & $21,6 \pm 6,1$ & $17,4 \pm 2,8$ & $23,7 \pm 6,6$ \\
\hline \multicolumn{5}{|l|}{ Primeras drogas utilizadas } \\
\hline Opiáceos & $65(54,6)$ & $23(95,8)$ & $17(94,4)$ & $7(46,6)$ \\
\hline Cocaína "crack" & $2(1,7)$ & $1(4,2)$ & 0 & $4(26,6)$ \\
\hline Cocaína & $2(1,7)$ & 0 & 0 & 0 \\
\hline Anfetaminas & $37(31,1)$ & 0 & 0 & $3(20)$ \\
\hline LSD & $4(3,4)$ & 0 & 0 & 0 \\
\hline Solventes & $1(0,8)$ & 0 & 0 & $1(6,7)$ \\
\hline Benzodiacepinas & $8(6,7)$ & 0 & $1(5,6)$ & 0 \\
\hline \multicolumn{5}{|l|}{ Modo de administración } \\
\hline Intravenosa & $43(41,2)$ & $2(8,3)$ & $12(66,7)$ & $2(13,3)$ \\
\hline Fumada & $8(9,2)$ & $19(79,2)$ & $1(5,6)$ & $6(40)$ \\
\hline Oral & $36(47,2)$ & $3(12,5)$ & $5(27,7)$ & $6(40)$ \\
\hline Inhalación & $2(2,5)$ & 0 & 0 & $1(6,7)$ \\
\hline Nunca inyectada & $81(68,1)$ & $2(8,3)$ & $18(100)$ & $5(33,3)$ \\
\hline $\begin{array}{l}\text { Nunca comparte } \\
\text { jeringas o agujas }\end{array}$ & $47(39,5)$ & $2(8,3)$ & $15(83,3)$ & $1(6,7)$ \\
\hline Comparte jeringas & $4(J, 5)$ & $2(0,3)$ & $1 J(0 J, J)$ & $1(0, i)$ \\
\hline o agujas actualmente & $8(6,7)$ & 0 & $2(11,1)$ & 0 \\
\hline $\begin{array}{l}\text { Solicitaron asistencia } \\
\text { por ellos mismos }\end{array}$ & $59(49.6)$ & $15(62.5)$ & $13(72.2)$ & $5(33,3)$ \\
\hline Contactos con otras agencias & $59(49,6)$ & $8(33,3)$ & $8(44,4)$ & $10(66,6)$ \\
\hline Desempleo & $112(94,1)$ & $24(100)$ & $18(100)$ & $15(100)$ \\
\hline Acomodación estable & $52(43,7)$ & $22(91,7)$ & $4(22,2)$ & $6(40)$ \\
\hline Guarda relaciones familiares & $25(21)$ & $18(75)$ & $1(5,5)$ & $8(53,3)$ \\
\hline Dificultades legales & $45(37,8)$ & $3(12,5)$ & $5(27,8)$ & $1(6,7)$ \\
\hline Antecedentes de prisión & $57(47,9)$ & $10(41,7)$ & $11(61,1)$ & $5(33,3)$ \\
\hline
\end{tabular}


Con el fin de evaluar los resultados de este estudio, se han utilizado tres análisis separados aplicando el Chi cuadrado. Uno para comparar el grupo asiático con el de británicos de origen caucásico. El segundo para comparar el colectivo afro-caribeño con el de británicos de origen caucásico y el tercero, para comparar el grupo de irlandeses con los británicos de origen caucásico.

\section{Modelo de consumo asiático}

La muestra de asiáticos contaba con una edad media $( \pm \mathrm{SD})$ en el momento de la presentación de 24,5 años $( \pm 4,4)$ mostrando grandes diferencias con la de los británicos de origen caucásico $(\mathrm{p}<0,0001)$ cuya edad media era de 32,2 años $( \pm 7,5)$. No existían diferencias importantes en cuanto a la edad en la que se comenzó a consumir droga, que para el grupo de asiáticos era de 21,6 años $( \pm 4,2)$ y de $21,3( \pm 6,1)$ para el grupo de británicos de origen caucásico.

La droga que más se consumía no era la misma para todos los grupos y, en el caso de los asiáticos se observó un alto consumo de opio $(95,8 \%$ Vs $54,6 \%$ de los británicos de origen caucásico; $\mathrm{x}^{2}=15,344$, df $=3, \mathrm{p}<$ 0,001). La forma en la que se administraba la droga era diferente en cada grupo $\left(\mathrm{x}^{2}=\right.$ $58 / 972$, df $=3, \mathrm{p}<0,001)$. El grupo de asiáticos mantenía una tasa de tabaquismo sensiblemente más alta que el resto $(79,2 \% \mathrm{Vs}$ $9,2 \%$ ). Sin embargo, este grupo ofreció un porcentaje mucho menor de utilización de la vida parenteral $\left(8,3 \%\right.$ Vs $68,1 \%, \mathrm{x}^{2}=$ $29,262$, df $=1, \mathrm{p}<0,001)$ así como de compartir agujas y jeringuillas (8,3\% Vs $39,5 \%$; $\left.\mathrm{x}^{2}=8,611, \mathrm{df}=1, \mathrm{p}>0,01\right)$. La proporción de compartir agujas y jeringas entre los dos grupos es muy parecida.

Aunque existían un amplio porcentaje de miembros del grupo asiático que acudieron por sí mismos a los Servicios (el 62,5\% Vs el 49,6\%), las diferencias no alcanzaron un nivel que fuese digno de ser mencionado $\left(\mathrm{x}^{2}\right.$ $=1,335$, df $=1, \mathrm{p}<0,5)$.

Una pequeña proporción del grupo de asiáticos contactó con otras agencias (el 33,3\% Vs el 49,6\%) pero la diferencia no resultó ser significativa $\left(\mathrm{x}^{2}=2,117, \mathrm{df}=1, \mathrm{p}<0,5\right)$.

Al comparar las variables sociales y legales del grupo de personas asiáticas y el de las británicas de origen caucásico, se observó que los asiáticos alcanzaban cotas de desempleo superiores aunque sin llegar a un nivel preocupante (el 100\% Vs $94,1 \% ; x^{2}=1,484$, df $=1, \mathrm{p}<0,5)$. A pesar de ello, eran los miembros de este grupo los que tenían vivienda fija $\left(91,7 \%\right.$ Vs $43,7 \%$; $x^{2}=18,405$, df $=\mathrm{p}<0,001)$ y menos problemas de tipo legal $\left(12,5 \%\right.$ Vs $37,8 \% ; x^{2}=5,740, d f=1, p$ $<0,02)$. En cuanto a los antecedentes penales, no se observaron diferencias dignas de mención $\left(\mathrm{x}^{2}=0,404, \mathrm{df}=1, \mathrm{p}<0,5\right)$.

\section{Modelo de consumo de los afro- caribeños}

La muestra compuesta por personas de origen afro-caribeño tenían una media de edad $( \pm$ SD) de 31,2 $( \pm 6,8)$ en el momento de la presentación. En cuanto al primer contacto con la droga la media se situaba en los 23,7 años $( \pm 6,6)$. Estas edades eran parecidas a las del grupo de británicos de origen caucásico.

El tipo de droga más importante que consumían los afro-caribeños no era igual a la de los británicos de origen caucásico $\left(\mathrm{x}^{2}=\right.$ $14,769$, df $=3, p<0,01)$. La forma de administración de la misma tampoco coincidía $\left(\mathrm{x}^{2}=13,556, \mathrm{df}=3, \mathrm{p}<0,01\right)$. El primero de estos grupos mostraba altas tasas de consumo de cocaína $(26,7 \%$ Vs $1,7 \%)$ y de tabaco $(40 \%$ Vs $9,2 \%)$. Sin embargo, las 
proporciones disminuían considerablemente en lo que a administración parenteral respecta $\left(33,3 \%\right.$ Vs $68,1 \% ; x^{2}=13,462, \mathrm{df}=1$, $\mathrm{p}<0,001)$ y al hecho de compartir agujas y jeringuillas $\left(6,7 \%\right.$ Vs $39,5 \% ;\left(x^{2}=6,2, d f=\right.$ $1, \mathrm{p}<0,02)$. En lo que a compartirlas ahora respecta, no existían diferencias significativas $\left(\mathrm{x}^{2}=1,072, \mathrm{df}=1, \mathrm{p}<0,5\right)$.

Las diferencias entre ambos grupos en cuanto al hecho de acudir por propia decisión, eran mínimas $\left(\mathrm{x}^{2}=1,409, \mathrm{df}=1, \mathrm{p}<\right.$ $0,5)$ así como en cuanto al contacto con otras agencias $\left(x^{2}=0,165, d f=1, p<0,5\right)$. Tampoco fue posible marcar distinción alguna en el apartado del desempleo $\left(\mathrm{x}^{2}=0,074, \mathrm{df}=\right.$ $1, \mathrm{p}<0,5)$. Por otro lado los afrocaribeños mostraron un alto nivel de contactos con la familia $\left(x^{2}=7,499 ; d f=1, p<0,01\right)$. En otro orden de cosas, los afro-caribeños no tenían problemas legales dignos de mención $\left(\mathrm{x}^{2}=\right.$ $5,733$, df $=1, \mathrm{p}<0,02)$ y no había diferencias entre los dos grupos en lo que a sus antecedentes de haber estado en la cárcel se refiere $\left(x^{2}=1,275, \mathrm{df}=1, \mathrm{p}<0,5\right)$.

\section{Modelo de consumo irlandés}

La muestra irlandesa tenía una media de edad $( \pm$ SD) en el momento de la presentación de 30 años $( \pm 6,8)$. El primer contacto con la droga se producía a los 17,4 años ( \pm $2,8)$, como media. Ambas edades eran parecidas a las que mostró el grupo de británicos de origen caucásico.

Existían diferencias importantes entre el primer tipo de droga que consumieron los irlandeses -que preferían el opio- y los británicos de origen caucásico $\left(\mathrm{x}^{2}=10,913, \mathrm{df}\right.$ $=3, \mathrm{p}<0,02)$. Aunque los métodos que utilizaban para administrase la droga eran similares, $\left(x^{2}=4,284\right.$, df $\left.=3, p<0,5\right)$, los irlandeses registraron mayor proporción de "nunca inyectarse" (100\% Vs 68,1\%; $x^{2}=$
$7,954$, df $=1, \mathrm{p}<0,01)$ y nunca compartir "agujas y jeringuillas" (83,3\% Vs 39,5\%; $\left(\mathrm{x}^{2}=12,128, \mathrm{df}=1, \mathrm{p}<0,001\right)$. No se encontraron diferencias significativas en cuanto a "compartir agujas y jeringuillas ahora" $\left(\mathrm{x}^{2}=0,445\right.$, df $\left.=1, \mathrm{p}<0,5\right)$.

A pesar de que había una alta proporción de individuos que acudieron por ellas mismos en el grupo irlandés, no se llegó a alcanzar un nivel importante $(72,2 \%$ Vs 49,6\%; = $3,215$, df $=1, p<0,5)$. No se observaron diferencias dignas de mención al comparar las variables sociales y legales entre el grupo de irlandeses y el de británicos de origen caucásico.

\section{Discusión}

\section{Consideraciones metodológicas}

Al estar centrados en un equipo comunitario de drogodependencias de tipo restrictivo quedan excluidos los casos menos importantes y aquellos otros que no tenían contacto con ningún Servicio de Toxicomanías, así como aquellos consumidores de drogas muy enfermos y aquellos que están hospitalizados o se encuentran en Centros de Tratamiento de Toxicomanías. Por otro lado el origen retrospectivo de este estudio limita el grado de exactitud de los datos recogidos. También la recopilación de estos datos se basó en los que estaban registrados y por tanto, dependía de algún modo de las evaluaciones que efectuaron otras personas así como de los diagnósticos clínicos. Por otra parte, al ser un mismo psiquiatra el que valoraba a los pacientes en diferentes ocasiones, ello garantizaba el grado de validez del examen. Si bien la evaluación del psiquiatra estaba clínicamente orientada, no existía información multidisciplinar inde- 
pendiente como datos sobre conductas de riesgo, sobre adaptación social o sobre comportamientos criminales. Por tanto, sólo se examinó un número limitado de variables.

Aunque la amplitud de la muestra es considerable, cuando queda dividida en grupos étnicos, los subgrupos resultan más pequeños. De este modo, se ha tenido que reunir a personas de etnias asiáticas diferentes pero que pertenecían a la misma raza (india y pakistaní). La representación de mujeres en este estudio es muy pobre. Finalmente, es importante destacar como una de las limitaciones de este estudio es que al ser descriptivo, no establece relaciones causales entre las variables.

\section{Modelo asiático}

Las personas de origen asiático se presentaron a los Servicios antes que los británicos de origen caucásico y un alto porcentaje de ellos lo hicieron por decisión personal. Estos descubrimientos contradicen a los de Oyefes y Ghodse (1994) según los cuales los bebedores asiáticos de riesgo evitan solicitar los servicios de la Atención Primaria para no ser clasificados peyorativamente de acuerdo con las actitudes prevalecientes en su medio cultural.

Puede que nuestros hallazgos se deban a la especial situación del Centro Baker Street, en el que se concentra un gran número de personas asiáticas, además la diferenciación cultural, sería cierto en el caso del alcohol, no así en el de las drogas.

\section{Modelo afro-caribeño}

Las personas de origen afro-caribeño mostraron un amplio porcentaje de consumidores de cocaína, lo que confirma los hallazgos de Kandal et al., según los cuales las personas de raza negra y los hispanos presentan una tendencia más acusada a tomar cocaína que los blancos. Sin embargo, esta afirmación contradice los estudios de Shaffer et al., para quienes el consumo de cocaína estaba más relacionado con las actividades delictivas entre consumidores de droga de raza blanca y negra. Nuestra muestra dio cuenta de un porcentaje muy reducido de problemas legales (entre los afro-caribeños).

Probablemente, esto sea debido a la diferencia que existe entre las dos muestras, la de Shaffer et al. estaba formada por adictos que habían sido arrestados por el Departamento de Policía de Baltimore en alguna ocasión, en cambio la nuestra está formada por una muestra comunitaria menor.

Un amplio porcentaje de las personas de origen asiático y africano-caribeño mantenían sus relaciones familiares y poseían una vivienda. En el caso de los asiáticos, estas actitudes se deben a que los núcleos familiares están formados por muchos miembros y ello puede funcionar como un factor que previene los problemas de tipo legal.

\section{Modelo irlandés}

Un alto porcentaje de los miembros del grupo irlandés habían acudido por sí mismos y algunos de ellos habían entrado en contacto con agencias pudiendo ello estar relacionado con el difícil acceso que los toxicomanos tenían a los servicios en Irlanda. Sin embargo, Riordan (1994) informó de cómo los pacientes acudieron al PIAA (Positively Irish Action on AIDS) informándoseles sobre los servicios disponibles así como sobre la forma de utilizarlos. La comunicación cara a cara y a viva voz es característica de la sociedad irlandesa y los datos monitorizados indican que de este modo, se llega a la mayoría de pacientes. Según el sistema 
monitorizado del PIAA el 53\% de los pacientes habían acudido al PIAA por ellos mismos, el $41 \%$ fueron enviados por agencias y el $6 \%$ por algún miembro de su familia.

El hecho de que los toxicómanos tuvieran acceso mediante el contacto verbal con tanta facilidad está relacionado con aspectos de la migración específicos de este grupo. Además, ya se ha establecido que este grupo proviene de ambientes empobrecidos. Muchos de ellos han recibido una pobre educación y durante las entrevistas se observaron problemas de analfabetismo. Así para estas personas, la comunicación verbal es una forma más efectiva de acceder a la información que los panfletos o los posters (O’Brien y Power 1998).

El consumo de opio y la temeridad de inyectarse utilizando jeringas compartidas o utilizadas por el grupo irlandés, en nuestra muestra, fue muy superior a la del grupo británico. Esta afirmación está relacionada con el informe presentado por el equipo de investigación sobre inmigrantes irlandeses afectados por el VIH y el SIDA (O'Brien y O’Brien 1998). El perfil de riesgo mostraba que el $100 \%$ de los individuos se había inyectado en alguna ocasión, según los informes de 1994/95 y 1995/96, el 64\% en los últimos 12 meses (1995/96) y el 49\% en 1994/95. Con el fin de comprender la conducta de riesgo de los consumidores de drogas irlandeses en Gran Bretaña, es importante conocer cómo afecta la identidad irlandesa a estas conductas de riesgo.

En Irlanda, hasta hace relativamente poco tiempo, la Iglesia gozaba de gran aceptación e influencia. La ideología católica ha contribuido en gran medida a la percepción del sentimiento de culpa en torno a los conceptos de "correcto" e "incorrecto", que impregnan profundamente a la sociedad irlandesa. Dentro del orden católico, hacer algo que desde que uno es niño se sabe que está mal, puede ser perdonado si, por ejemplo, se han tomado algunas copas. En cambio, la premeditación de un acto es algo mucho más grave implicando un mayor grado de culpabilidad. El hecho de tener una jeringuilla limpia indicaría que la acción ha sido premeditada y por tanto que se trata de un pecado según la doctrina católica; de ahí que surjan conflictos relacionados con los mensajes educativos para la salud que tengan como objetivo la reducción de estas conductas de riesgo (O’Brien y Power 1998).

Aunque el criterio físico más sencillo para llevar a cabo una clasificación social puede ser el color de la piel, las personas que pertenecen a una misma categoría racial pueden, no obstante ello, pertenecer a diferentes grupos étnicos $\mathrm{y}$, por tanto poseer valores culturales que den lugar a conductas diferentes (Riordan 1994). En nuestra muestra observamos diferencias de comportamiento relacionadas con el consumo de drogas entre los dos grupos caucásicos, el británico y el irlandés. Esto refrenda la teoría de Cheung (1993) sobre las diferencias de comportamiento en un mismo grupo en el que todos los miembros son de la misma raza, aportando las diferencias culturales más información. La población irlandesa posee una identidad basada en una serie de experiencias históricas que son diferentes a las que pueda tener la población británica. A pesar de la proximidad geográfica y de todos los permisos de residencia y de trabajo que tienen los irlandeses, el proceso de emigración no es fácil (O’Brien y Power 1998). Se ha aceptado que esta proximidad geográfica y cultural de Irlanda y Gran Bretaña, el poseer la misma lengua y sobre todo el mismo color de piel, haría a este grupo inmune a las diferencias de sus niveles de salud por parte de grupos minoritarios y emigrantes (Allen 1994). Estos resultados 
muestran que es necesario fomentar las actividades de prevención y lograr que los grupos étnicos minoritarios tengan acceso al mundo del trabajo, así como que los Servicios que se implanten cuenten con las dificultades que tienen muchas veces estos colectivos para llegar a contactar con las Instituciones asistenciales oficiales. Del mismo modo se deberían facilitar a estas personas el acceso a determinados aspectos prácticos tales como la vivienda y el trabajo.

\section{Bibliografía}

ALLEN, T.W. The invention of the White race. Vol. one: Racial oppression and social control, London: Verso, 1994.

ANGLIN, M.D., RYAN, J.M., BOOTH, M.W., HSER, Y. Ethnic differences in narcotics addiction: I characteristics of Chicano and Anglo methadone maintenance clients. Int J Addict 23(2): 125-149, 1988a.

ANGLIN, M.D., RYAN, J.M., BOOTH, M.W., HSER, Y. Ethnic difference in narcotics addiction: II Chicano and Anglo Addiction career pattern. Int J Addict 23(10): 10111027, 1988b.

BACHMAN, J.G., JOHNSON, O'MALLEY, P.M. Monitoring the future: Questionnaire responses from the Nation's High School Seniors 1986. Ann Arbor MI: Survey Research Centre, Institute for Social Research, University of Michigan, 1987.

BIRCHWOOD, M., COCHRANE, R., MACMILLAN, F., et al. The influence of ethnicity and family structure on relapse in first-episode schizophrenia, a comparison of Asian, Afro-Caribbean and White patients. Brit. Psychiat 161: 783-790, 1992.

CATALANO, R.F., HAWKINS, J.D., KRENZ, C., et al. Using research to guide culturally appropriate drug abuse prevention. J Consult Clin Psychol 61:804-811, 1993.

CHEUNG, W. Approaches to ethnicity. Clearing road block in the study of ethnicity and substance use. The Int $J$ Addict 28(12): 1209-1226, 1993.

CHEUNG, W. Ethnicity and alcohol/drug use revisited: a framework for future research. The Int J Addict 25 (5A \& 6A): 581-605, 1990-1991.
COCHRAN, R. Mental illness in immigrants to England and Wales: an analysis of mental hospital admissions, 1971. Social Psychiatry 12: 25-35, 1977.

HWU, H., CHEN, C., YEH, L. A typology of alcoholism in Taiwan Aborigines: a study on evolving process of community cases. Psychopathology 25: 311-318, 1992.

KANDEL, D., SINGLE, E., KESSLER, R.C. The epidemiology of drug use among New York High School students: distribution, trend and change in rate of use. Am J Publ Health 66: 43-53, 1975.

KARON, M., JENKINS, J.H., DE LA SELVA, A., et al. Expressed emotion and schizophrenic outcome among Mexican American families. J Nervous \& Mental Disease 175: 143-151, 1987.

LIEBERSON, S. Unhyphenated Whites in the United States. Ethn and Rac Stud 8: 159-180, 1985.

LIPSEDGE, M., DINAN, G., DUCKWORTH, E. A preliminary survey of Italian intravenous heroin users in London. Addiction 88: 1565-1572, 1993.

MARSHALL, G. Oxford concise dictionary of sociology, Oxford. Oxford University Press, 1996.

McKEIGUE, P.M., KARMI, G. Alcohol Consumption and Alcohol Related Problems in Afro Caribbean and South Asia in the United Kingdom. Alcohol 20: 1-10, 1993.

O'BRIEN, O., POWER, R. HIV and a Migrant community: they Irish in Britain. London. Action Group for Irish Youth, AGIY, 356 Holloway Road, 1998.

OYEFESO, A., GHODSE, A.C. Addictive Behaviour in ethnic minorities: In Oyefeso A.C. Sociocultural aspects of substance use and misuse. Current Opinion in Psychiatry 1993; 87: 273-277, 1994.

RIORDAN, S. Evaluating a community-Based Initiative. The Drug and Irish Mobility Project. London. Positively Irish Action on AIDS, 1994.

SHAFFER, J.W., NUTCO, D.N., BALL, J.C., KINLOCK, T.W. The frequency of non-narcotic drug use and its relationship to criminal activity among narcotic addicts. Comp Psychiat 26: 558-566, 1985.

Dirección para correspondencia:

Dr. Hamdy F. Moselhy MBBCh, Msc, DPM, MRCPsych,

Psiquiatra

Centro de Conducta Adictiva, Highcroft Hospital,

71 Fentham Road Erdington,

Birmingham

B23 6AL

REINO UNIDO 